\title{
ATM Network Resources Management using Layer and Virtual Network Concepts
}

\author{
G. Woodruff, N. Perinpanathan, F. Chang, P. Appanna, A. Leon-Garcia \\ Department of Electrical and Computer Engineering, University of Toronto \\ Toronto, Ontario, M5S 1A4. Canada \\ Phone: (416) 978-4764 Fax: (416) 978-4425 \\ gillian@comm.toronto.edu / appanna@comm.toronto.edu /alg@comm.toronto.edu.
}

\begin{abstract}
This paper describes the organization of a resource management system for an ATM network within an integrated operations environment. The system encompasses both traffic and network management aspects within a single framework. A management hierarchy is described based on the concept of layer networks, and a new Virtual Network layer is defined for fairly allocating network resources amongst competing network and service applications. Each Virtual Circuit (VC), Virtual Path (VP), Virtual Network (VN) and Transmission Path (TP) layer network is assigned resources which can be accessed by and assigned to other client layers where applicable for the routing and admission of transport connections. Examples of general layer network management entities and an object-oriented information model capturing required resource and performance information are presented to illustrate the power of re-using concepts at each management layer.
\end{abstract}

Keywords: Virtual Network, Layer Network, Resource Management, Information Model.

\section{INTRODUCTION}

One of the key transport technologies within the future broadband network will be ATM (Asynchronous Transfer Mode). ATM standards include aspects related to connection control and network management. There is also now an enormous body of theoretical literature addressing related ATM traffic and resource management issues and requirements, some of which are far from resolved; and although their algorithmic aspects would not be subject to standardization, the transport network operator's connection control and management system must have access to a standard set of relevant network state information.

The objective of this paper is to bring together what we perceive as some essential elements of an ATM Network Resources Management (NRM) system within a management framework encompassed by standards bodies and consortia. Our initial focus here is on a system which is to operate within a single administrative domain of a public network. An NRM system, in our definition, is responsible for all aspects related to the fair allocation of transport network resources to competing users; as such, it includes: i) traffic management (ATM cell-level flow controls and connection set-up/release); and ii) network management (performance monitoring and configuration management). Controls of type i) would traditionally be proprietary and fall under the responsibility of the network access and switching equipment, whereas ii) is traditionally the responsibility of the network management system, with standardized interfaces now being defined within the ATM Forum and the ITU-T's Telecommunications Management Network (TMN) [M.3010].

The Telecommunications Information Networking Architecture Consortium (TINA-C) provides the framework for developing these traditionally separate NRM controls on a common object-oriented software platform in a distributed processing environment, allowing the sharing of common network resource information and management functionality. In this paper we give an overview of a possible 
NRM architecture for ATM, based on concepts from TMN, TINA-C, the ATM Forum, and current ATM traffic management research. We start in Section 2 with a summary of ATM's layer networks and in Section 3 with an overview of what resources are to be managed and how these resources can be organized using the layer network and Virtual Network concepts. The virtual network is a logical collection of switching and transmission resources, and simplifies management of these resources. This is followed by an overview of the NRM architecture in Section 4, the managing entities required to support basic NRM functionality in Section 5, and some essential elements of the NRM Information Model to represent resource states in Section 6.

The objective is to define general concepts of ATM resources and NRM management functions which can be re-used at different levels of a management hierarchy to provide a rich and versatile "toolkit" applicable to a wide range of NRM needs. This effort is part of a larger project whose aim is to develop an integrated resource management architecture for large-scale, integrated services ATM networks. There are five projects across several Canadian universities addressing different facets of the larger project.

\section{ATM CONNECTION AND LAYER NETWORKS}

ATM has the ability to support two types of connections (referred to as "trails" by ITU-T and TINAC): Virtual Circuit connections (VCCs) and Virtual Path connections (VPCs). Each VCC/VPC consists of a concatenated set of VC/VP links (VCLs/VPLs) connected by nodes supporting VC/VP switching. Each VC/VP link makes use of an identifier (VCI/VPI) which is used for switching and is translated into the identifier for the next link. VCLs can (but need not) be carried within VPCs; this simplifies VC connection set-up procedures, as less VC switches need be involved, augments the connection-carrying capacity of the network (limited by VCI address space), and provides a means to partition groups of like users.

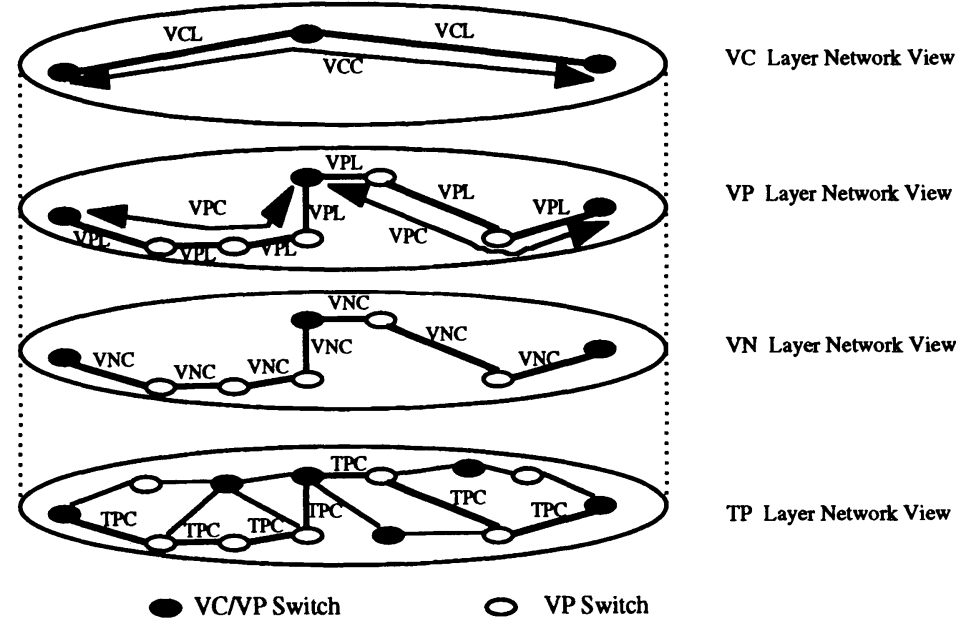

Figure 1: The NRM layer networks.

The layer network concept [G.805][TINA95] can be applied to ATM to view the connection topology and resources at the VC, VP and Transmission Path (TP) layers, as shown in Figure 1. The VP layer is considered a server to the VC layer; the TP layer can be considered a server to both the VP layer and the VC layer (when VCCs are not routed through VPCs). In the NRM context, a server layer can allocate some of its resources to the client layer (e.g. some transmission capacity assigned to a 
server VPC can be allocated to a client VCL). In addition, a server layer can define the restricted topology over which routing procedures may be carried out for a client layer connection, e.g. a VCC could be routed over the server VP topology, making use of information regarding VPC resource availability.

The layer network concept is an important one for the NRM system, as each layer provides a simplified logical network view of a specific portion of network resources, within which management functions can be carried out. We have extended the layers to include a Virtual Network (VN) layer, as illustrated in Figure 1 and motivated in the following section.

\section{THE NEED FOR RESOURCE MANAGEMENT: ATM SERVICE GUARANTEES}

\subsection{Levels of resource allocation and Virtual Networks}

A resource management system is necessary due to the need to allocate limited resources amongst competing users and to monitor resource usage. In the ATM transport network, competition for resources will occur at several levels.

At the cell level, VC or VP connections being multiplexed or switched at Network Elements (NEs) compete for buffer space and transmission time and consequently may experience cell loss and variable cell transit delays; faimess can be judged by providing cell-level Quality-of-Service (QoS) guarantees for each connection, and is realized through the Usage Parameter Control (UPC) or Network Parameter Control (NPC) of agreed connection traffic flow characteristics (Traffic Descriptors or TDs), buffer management, and scheduling policies at these NEs.

A large body of the ATM literature has focused on the development of models relating these policies and the cell-level QoS, which depend on the traffic flow characteristics, buffer and bandwidth resources, and switching architecture. We believe that a standard "black box" representation of specific switch and multiplexer performance capabilities is necessary if connection management functions are to be unbundled from the switching elements. This representation would be used to determine whether a new connection with specific TDs and QoS needs can be supported. From the resource management perspective, it is highly desirable to transpose this procedure into one where the NE capabilities and the connection requirements can be represented as a set of additive "virtual" resources to be allocated or released.

The equivalent bandwidth concept is one promising approach [ElWa193][Kesid93] in which the common resource "currency" is transmission bandwidth. The development of other capacity representations which account for multipoint switching or specific scheduling implementations with limited buffering remains an open issue. For our purposes here, we assume that an NE is capable of supporting a connection's TD and QoS requirements when the required equivalent bandwidth can be allocated to the associated terminating VCL or VPL. This provides a convenient link-oriented representation of NE resources required to provide cell-level QoS, which can be assigned to server layer networks and allocated amongst their clients.

At the connection (VC or VP) level, requests for connectivity may be denied due to a lack of NE resources to support cell-level QoS requirements (e.g. equivalent bandwidth), or due to a lack of VCI/VPI addresses or other resources such as processing required to perform per-connection UPC, flow control or scheduling, and buffer management; fairness at this level can be judged by providing connection-level Grade-of-Service (GoS) guarantees such as probability of blocking, and is realized through the "virtual" dedication of pools of these resources throughout the network to designated classes of users making connection requests.

We refer to this subset of network resources for a class of users as a Virtual Network or VN; within this network, requests for VC or VP connectivity or an increase in their resource allocations may be treated on an equal, first-come-first-served basis or on some other scheduling schemes. This concept of VN is more general than that of a collection of VPCs, as it provides a means for fair allocation of total TP layer resources amongst competing classes of transport applications. The resources allocated logically to the VN are managed as a set. All the VPCs, VCCs inside a VN may be managed as one entity instead of individually. Instead of a VN, if we use a collection of VPCs, then each VPC has to be managed or monitored singly as opposed to a group. Additionally, allocating resources to the VN will 
simplify resources management as now, only a subset of the network resources is to be managed as opposed to the entire set of network resources. For example, if VNs are defined for a particular class of traffic, the allocation/de-allocation of resources are eased because it is much simpler to allocate resources to similar traffic (homogeneous) than heterogeneous traffic. The logical segregation of resources will increase blocking probability but this is counterbalanced by multiplexing gain that are produced by the sharing of resources by the homogeneous traffic of the VN. For the large-scale network under consideration, the multiplexing gain should be significant.

The VN concept has only lately been appearing in the ATM literature under various nomenclature [Dziong96][Farago95], and we believe it will be a powerful and versatile resource management tool for large-scale networks. For example, VNs may be dedicated for a particular Q0S/service/traffic class (e.g. video traffic), for an overlay network dedicated to a particular application (e.g. IP overlay network), or for a particular customer group with specific network management requirements (e.g. as a faultprotected Virtual Private Network); VNs could be nested within other VNs; and significant gains in bandwidth efficiency are possible by utilizing VP connections for fast routing only, with VC connection resources drawing upon the total VNC (see definition below) resource pools rather than individual VPC [Dziong96].

For this reason, we have added an additional VN layer network between the VP and TP layers in Figure 1. This Figure illustrates the physical topology of a VN, which corresponds to that of the TP layer. Since the VCCs and VPCs resources are drawn from the VN, it is logical to put the VN layer between the TP and the VP layer, instead of between other layers. We adopt the same layer network terminology, such that VPCs at a client VP layer are routed over Virtual Network connections (VNCs) at the VN layer. VNCs are shown routed in a "single hop" over the TP topology (and therefore consist of a single VN link - VNL); each VNC is assigned a portion of the TP connection's (TPC's) equivalent bandwidth. We assume that each VNC is also allocated a pool of VCI/VPI addresses to be associated with VCL/VPL terminations. Other NE resources, such as processing, are also allocated to switches at each VN layer.

Note that the routing over a VN (its logical connectivity) can be further restricted by defining a VN routing topology. This would imply a representation where VNCs are routed over multiple hops of the TP topology. However, since switching only occurs at VC and VP levels, no actual connectivity at the VNC level would be implied. The physical VN representation is necessary to capture information regarding the location of VC/VP switching nodes and resources associated with switching and terminating VCL/VPLs.

At the VN layer, there will also be competing requests for the limited resources of the TP (physical) network; it is therefore possible for VN requests to be denied. The way that fairness is judged at this level, and the associated sharing policies, are open research topics. However, all layer networks can be seen to share the pattern of connectivity requests (for a specified QoS and TDs) which are satisfied by allocating server layer resources. These resources need to be provisioned in such a manner as to meet the layer network's service guarantees. This repeating pattern motivates our definition of general layer network resource management functional entities discussed in Section 5.

\subsection{QoS management per Virtual Network}

The management of multiple QOS classes can be handled by establishing a VN for each class, and for illustrative purposes in this paper we will assume this approach. Resources allocated to a VN are then at the disposal of all connections which will be satisfied by the VN's QoS constraints. This can be achieved by establishing an end-to-end QoS reference connection and allocating QoS limits to each NE switch and link; a link's QoS allocation then equals its server layer's end-to-end connection QoS.

For example, a VPC would be routed over the VN layer network which supports the appropriate QOS class. The end-to-end QOS of the VPC would be the "sum" of the component QoS values associated with the NEs performing VP switching and the VNC "hops", and can be bounded by limiting the number of hops allowed over the VN layer. Similarly, a VCC routed over the VP layer would have an end-to-end Q0S equal to the "sum" of the component Q0S values associated with the NEs performing VC switching and the VPC "hops". The VCC end-to-end QoS can be similarly bounded by restricting the number of hops over the VP layer. 


\section{THE NRM ARCHITECTURE}

The NRM architecture consists of a structured set of interacting managing systems to realize real-time traffic control and network management. The architecture is aligned with TINA's functional layering, and the NRM system resides within TINA's resources layer and TMN's Network Management Layer (NML) and Element Management Layer (EML). The architecture captures the strong interactions among the managing systems to perform admission control, routing, resource allocation, resource reconfiguration, connection set-up/release, and performance monitoring functions necessary to effectively manage the ATM network's resources.

The managing systems that form the NRM architecture are shown in Figure 2. These managing systems are defined based on the computational modeling concepts specified by the TINA-C [TINA95a]. They include the Connection Session Manager, a set of Layer Network Managers (LNMs), and a set of Element Managers, which are organized and structured based on the ATM transport network functional architecture defined by ITU-T [I.326] and the management functional layering principle defined by TMN [M.3010]. Interactions among these managing entities consist of invocations, responses, and notifications.

Connection requests initiated at TINA's service layer, as depicted in Figure 2, are directed to the Connection Session Manager. The Connection Session Manager identifies the Virtual Network (VN) within which the connection is to be established, and directs the request to the appropriate LNM. The LMNs are defined for VC, VP, VN and TP layers, and perform dynamic resource management within their respective layer. For example, a VCC request with a particular QoS class is directed to the VC manager of the appropriate VN reserved for that Q OS class.

The Element Manager translates the physical NE state information into vendor-independent logical resource information for the LNMs. Each Element Manager is delegated an individual NE or a set of NEs. The Element Manager is notified by the NEs of faults, which are also polled to retrieve performance related parameters. Other functions of the Element Manager include: monitoring and reporting facility usage to the LNMs; interacting with local NEs to configure network connections upon the LNM's command; initiating continuity checks and traffic loop-back tests; and maintaining a local database to keep updated information about the local transmission path, link, and node states under its purview.

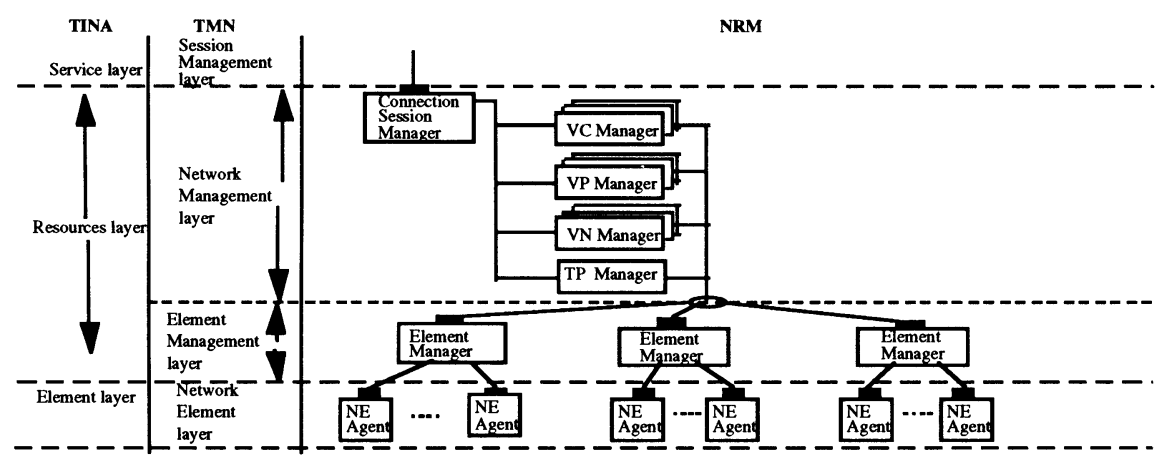

Figure 2: The NRM architecture.

\section{THE LAYER NETWORK MANAGER (LNM)}

Each Layer Network Manager performs similar traffic management and network management functions (such as connection management, resource reconfiguration management, and performance management) based on the resource states of its layer network. Therefore, to achieve high software re- 
use $^{1}$, it is desirable to define a generic LNM that can be specialized to yield a Virtual Connection (VC) Manager, Virtual Path (VP) Manager, Virtual Network (VN) Manager, and Transmission Path (TP) Manager. Note that a VC, a VP, and a VN Manager may exist for each Virtual Network defined over the Transmission Path layer network. However, only a single TP Manager is required irrespective of the number of VNs that are defined.

Such a generic LNM can be specified as a building block that consists of a set of managing entities, which in turn can be implemented using Computational Objects (COs) that interact with each other in order to make management decisions pertaining to the layer network. This approach is shown in Figure 3, which illustrates a generic LNM building block along with its managing entities and possible interactions (indicated by directed arcs), both within and external to the LNM. As shown in Figure 1, the VC layer network is considered the "highest" layer network while the TP layer network is considered the "lowest" layer network; therefore, lower-numbered LNMs can act as a server to any higher-numbered LNM and as a client to any lower-numbered LNM. For example, LNM(N) can act as a client to $\mathrm{LNM}(\mathrm{N}-1)$ and as a server to $\mathrm{LNM}(\mathrm{N}+1)$.

The managing entities defined within the generic LNM include the Admission Controller, the Routing Manager, the Resource Manager, the Connection Manager, the Configuration Manager, and the Network Information Manager. The Admission Controller, and the Configuration Manager act as clients to appropriate managing entities within lower-numbered LNMs. The Routing Manager and the Resource Manager act as servers to appropriate managing entities within higher-numbered LNMs. The Network Information Manager oversees the storage and monitoring of the layer's information base, which presents the necessary network state information for use by these managing entities.

Functions performed within each managing entity are transparent to the other managing entities. However, a managing entity may invoke another managing entity through appropriate interfaces to obtain its management services. Section 5.1 illustrates the sequence of interactions that take place among the above managing entities to set up a new VCC over the VP layer network. Section 5.2 then generalizes the functions performed by each of these managing entities so that they can be applied to a variety of network resource management tasks.

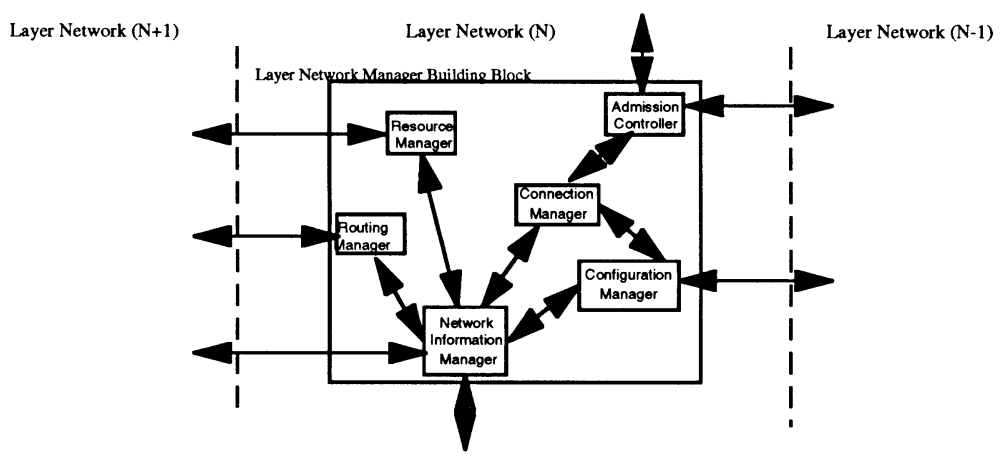

\subsection{Example: VCC set-up over VP layer}

Figure 4 shows the interaction among the managing entities within the VC, VP and VN Managers to set up a new VC connection. The Connection Session Manager sends a VCC request to the VC Admission Controller (1). The VC Admission Controller then invokes the server (in this case, the VP layer) to find an appropriate route over the server layer topology (2) and to reserve the necessary transmission

\footnotetext{
${ }^{1}$ Software re-use is achieved by instantiation of objects from the same object classes and by specialization that allows the addition of attributes and operations to an existing object to yield completely different objects. An object encompasses both function codes and data structures.
} 
resources (3). Switching resources at each VC switching point are reserved through invocation of the VN Resource Manager (4). The VC Admission Controller then invokes the VC Connection Manager (5) to perform the VC connection binding operations, and the VC Network Information Manager then instantiates the VCC and Termination Point objects within the VC layer's information base (6). The VC Network Information Manager then invokes the Element Managers for each VC switching node (7) to set the UPC parameters and the translation table entries with specified VCIs. Once the Network Information Manager receives a confirmation from the Element Manager, an acknowledgment is conveyed to the Admission Controller, which indicates to the Connection Session Manager that the new VCC has been set up.

Note that the set of interactions among the LNMs to set up a VPC over the VN layer network, or to set up a VCC over the VN layer network are similar to those shown in Figure 4. In the former case, the VP Manager acts as a client to the VN Manager, while in the latter case the VC Manager acts as a client to the VN Manager. This generality also extends to the case of setting up a VNC over the TP layer network.

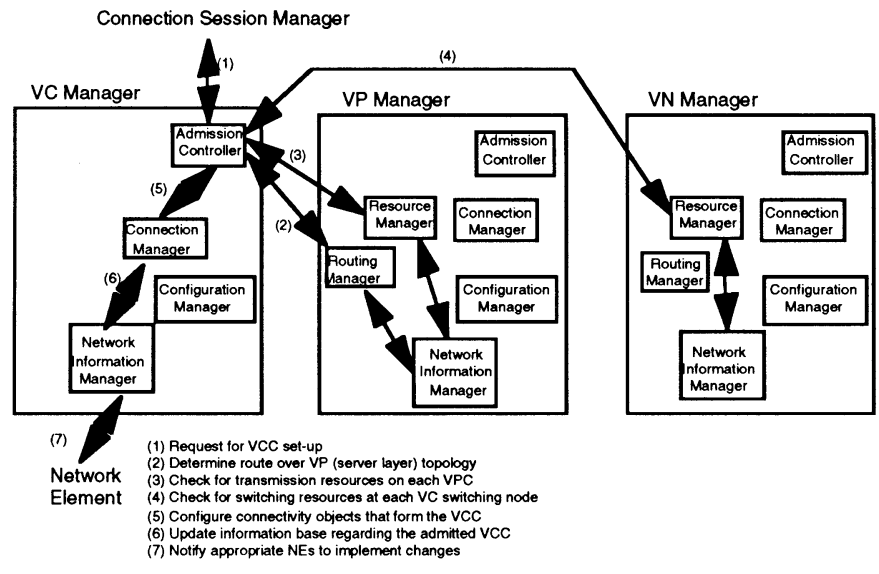

Figure 4: Interactions among the VC, VP and VN LNM managing entities

\subsection{Description of the LNM's generic managing entities}

This section gives a summary of the generic functions performed by each managing entity, and how these functions specialize at specific layers.

\section{Admission Controller}

The Admission Controller is invoked by the Connection Session Manager following a connection/topology request from the service layer, and coordinates the set of steps to decide whether a new connection/topology can be admitted into the network. A connection/topology specializes, for VC and VP layers, as a point-to-point or multipoint connection; for VN layers, as a network topology; and for the TP layer, as a "physical" network (which may itself be a layer network e.g. within SONET).

The Admission Controller interacts with the server Routing Manager and Resource Manager to determine whether a route and the necessary resources are available within the server layer network for connection/topology admission. Based on the responses received from these managing entities, the Admission Controller may decide to accept the connection/topology, reject the connection/topology, or optionally allow for re-negotiation in case of rejection. The number of rejected requests is stored by the Network Information Manager to track service levels being provided. 


\section{Configuration Manager}

The Configuration Manager is invoked internally, and coordinates a similar set of steps as the Admission Controller to dynamically modify the layer network's topology and resource states, either on a scheduled or on-demand basis, in order to track projected or sudden changes in traffic demand or topology. These functions specialize, for VC and VP layers, as changes in connection resource allocations, re-routing in the event of failure, or adding/dropping leaf terminations; for VN layers, as new resource allocations or topologies; and for the TP layer, as changes in switching nodes or transmission capacities within the ATM network domain. Changes in resource allocations may arise due to performance degradation at the specific layer (e.g. QoS degradation at the VC and VP layers, GoS degradation at the VP and VN layers, resource shortage at the TP layer) or due to dynamic adaptation to measured and projected traffic demand.

\section{Routing Manager}

The Routing Manager determines an appropriate route based on its layer network's topology and resource states (e.g. estimated or measured utilization of resources). The Routing Manager may be invoked by a client layer's Admission Controller during a new connection set-up, or by a client layer's Configuration Manager to perform re-routing in the event of failure or adaptive reconfiguration . The Routing Manager interacts with the Network Information Manager to obtain topology and resourcerelated information for route selection, which may in turn store a set of alternative routes for future use. Since routing is not performed at the VC layer network, a Routing Manager does not exist within the VC Manager.

\section{Resource Manager}

The Resource Manager regulates the allocation of its connection hop resources (such as effective bandwidth and addresses) and Network Element resources (such as processing and virtual resources) to satisfy the client layer's traffic demand and performance constraints. Network Element resources reside at VN and TP layers, while resources for a connection "hop" can reside at all layer views. The Resource Manager may be invoked to allocate or re-negotiate resources for a new connection, or to reassign resources allocated to already existing client layer connections. As with the Routing Manager, the Resource Manager does not exist within the VC Manager.

\section{Connection Manager}

The Connection Manager binds connection and Termination Point objects during connection set-up, and unbinds them during connection release. Functions performed by the Connection Manager include logical configuration of the switch, i.e. setting up translation table entries from incoming to outgoing ports. The Connection Manager may be invoked by the Admission Controller to bind a new connection, or by the Configuration Manager to bind a modified or restored connection. The Connection Manager in turn invokes the Network Information Manager to instantiate and update the set of objects that form the connection.

\section{Network Information Manager}

The Network Information Manager monitors the Management Information Base which captures the necessary topological and resource state information for layer management. Managing entities within a LNM invoke the Network Information Manager to update the network state information each time a management decision is made. The Network Information Manager in turn notifies the appropriate Element Manager(s) regarding changes in connections or resource states in order to physically effect the logical changes within the appropriate NEs. The Network Information Manager also receives performance-related parameters from the Element Manager. In addition, the Network Information Manager triggers the Configuration Manager to take appropriate actions whenever it detects a degradation in its layer network's performance.

The next section will discuss some aspects of the information model that are used by these managing entities for resource management. 


\section{NRM INFORMATION MODEL (NRM-IM)}

The NRM Information Model (NRM-IM) captures the necessary ATM network resource and performance information to enable the resource management functions. The NRM information model (NRM-IM) is an ATM-specific and protocol-independent information model that abstracts an ATM network into a logical view. This logical view contains all network architecture components, connection objects and related resource and performance information identified in previous sections of this paper. The NRM-IM establishes a standard view of the network among all managing entities so as to allow sharing of common network information.

The NRM-IM consists of two parts: a network view (nv) and an element view (ev). The element view focuses on modeling individual network elements, while the network view models the aggregation of the network elements and resources and provides a network-wide view. The element view Management Information Base (MIB) is used for communication between the NEs and the Element Managers at the EML. It is also used by the Element Managers at the EML to interact with the Layer Network Managers at the NML. The network view MIB is used for interactions among Layer Network Managers at the NML.

The NRM-IM (ev) is adopted from ATM Forum's M4 MIB element view [ATMF-M4ev] and Bellcore's TA-1114 [TA-1114]. The NRM-IM (nv) is based on ITU-Ts functional architecture of the transport network, both generic [G.805] and ATM-based [I.326], ATM Forum's M4 MIB network view [ATMF-M4nv], and TINA's Network Resource Information Model (NRIM) [TINA95b].

Figure 5 shows a subset of the NRM-IM (nv) object classes which contain the network resource and performance information for resource management. The object classes are specified using the widely used object model of Rumbaugh [Rumb91]. The object classes shown in this figure are generic classes. All object classes in each layer network inherit ${ }^{2}$ from these generic classes. For instance, the $\mathrm{Trail}$ class can be specialized ${ }^{3}$ to become a VCC, VPC, VNC and TPC in the VC, VP, VN and TP layer network respectively and the LinkConnection class can be specialized to become a VCL, VPL, VNL, and TPL. The inheritance and specialization principles of object-oriented technology enable the creation of objects that capture the attributes and operations at different abstraction level. They also allow code re-use. The Connection Termination Point (CTP) and the Trail Termination Point (TTP) are similar to the Network Connection Termination Point (NWCTP) and the Network Trail Termination Point (NWTTP) in TINA's NRIM, respectively.

The ATM network resource and performance information required for resource management is captured by the attributes of these object classes. The information or the attribute values would be gathered by assignment, estimation or measurement depending on the nature of the information. Network resources that we show are address space (VCI, VPI range), equivalent bandwidth and reserved switch processing. Parameters characterizing connections include traffic descriptors (TDs), QoS class and bandwidth utilization. Performance information is captured by the QoS performance parameters and GoS. These attributes are shown in Figure 5 and are listed below. Managing entities within each Layer Network Manager would manipulate these attributes while carrying out their corresponding resource management functions.

Address space -- Attribute of TTP.

The Address space can be a VCI range or VPI range or both. It indicates whether a VCI or VPI is available for a new connection.

Equivalent Bandwidth -- Attribute of Trail and LinkConnection.

This is an abstract representation of a connection's resource requirements at termination points based on the traffic descriptors and the QoS performance parameters of that connection.

Switch Processing -- Attribute of Subnetwork.

This is a quantitative representation of processing capacity available for a switch to handle new connections.

\footnotetext{
${ }^{2}$ Inheritance is the sharing of attributes and operations among classes based on a hierarchical relationship.

${ }^{3}$ Specialization: Each subclass inherits all the properties of its superclass and adds its own unique properties.
} 


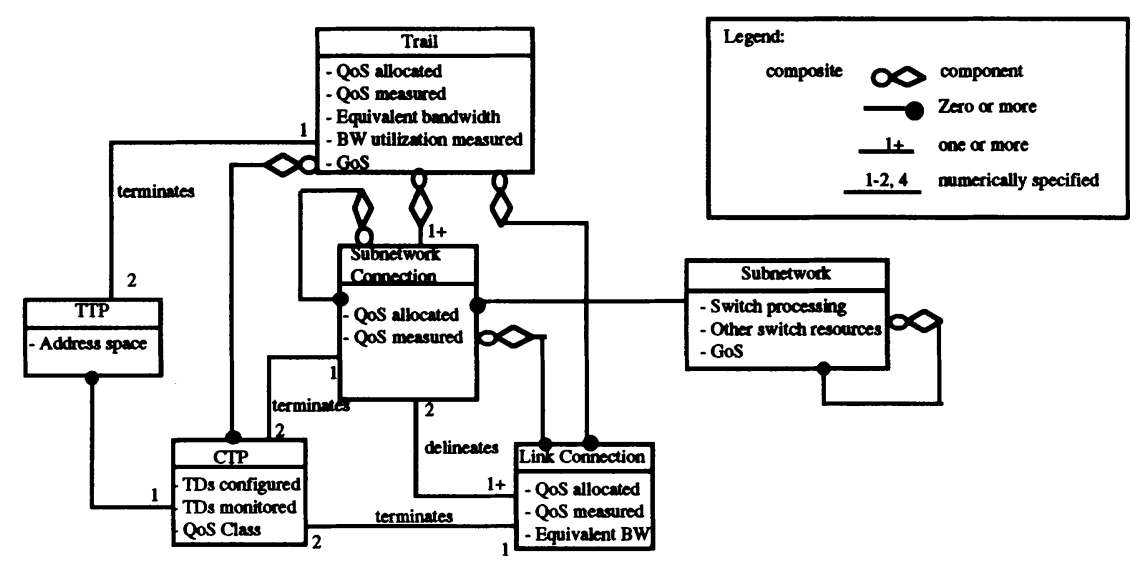

Figure 5: The NRM-IM (nv) object classes

Traffic Descriptors Configured -- Attribute of CTP.

This characterizes expected connection traffic flow. These TDs include Peak Cell Rate (PCR), Sustainable Cell Rate (SCR), Burst Tolerance (BT) and others, as defined by the ATM Forum and ITUT.

Traffic Descriptors Monitored -- Attribute of CTP.

This indicates the UPC/NPC violation counts and number of successfully passed cells at each CTP by aggregating the parameters obtained from the UPC/NPC Monitoring Data object class in the element view.

Bandwidth Utilization Measured -- Attribute of Trail

This reflects measurements of specific parameters describing actual traffic flow e.g. TDs, average and variance of cell rates, etc.

GoS -- Attribute of Trail.

This indicates the connection-level Grade of Service such as probability of blocking.

QoS Class -- Attribute of CTP.

The QOS Class can be specified or unspecified. Several QOS classes are specified by the ATM Forum to provide different types of services. A set of default values will be assigned to the QoS performance parameters if a specified QOS Class is assigned.

QoS -- Attribute of Trail, SubnetworkConnection and LinkConnection.

This is a set of QoS performance parameters that conveys the cell-level QoS performance information for the given connection segment. These parameters are defined by both the ATM Forum [ATMF-v4] and ITU-T [I.371]. There are two types of QoS performance parameters. The QoS allocated is the assigned QOS contribution allowed for that connection or connection segment, based on the assumed reference connection. The QOS measured is the actual cell-level performance obtained through measurement. For instance, the QoS performance values are typically measured by sending OAM cells intrusively or non-intrusively through the segment of interest and applying loopback at the far end. The values of these attributes would then be calculated by studying the received OAM cells. 
Typically, all objects are contained in either ATM NEs or layer networks. Although this provides a clean partitioning of the objects, object relationships that relate objects in different layer networks and views (ev/nv) are still required to maintain the integrity of the information model:

- Objects with inter-layer relationship: LinkConnection and Trail classes are involved in the inter-layer relationship. Based on the functional architecture of the transport network, a link connection at the client layer will be served by a trail at the server layer. The relationship is illustrated in Figure 6.

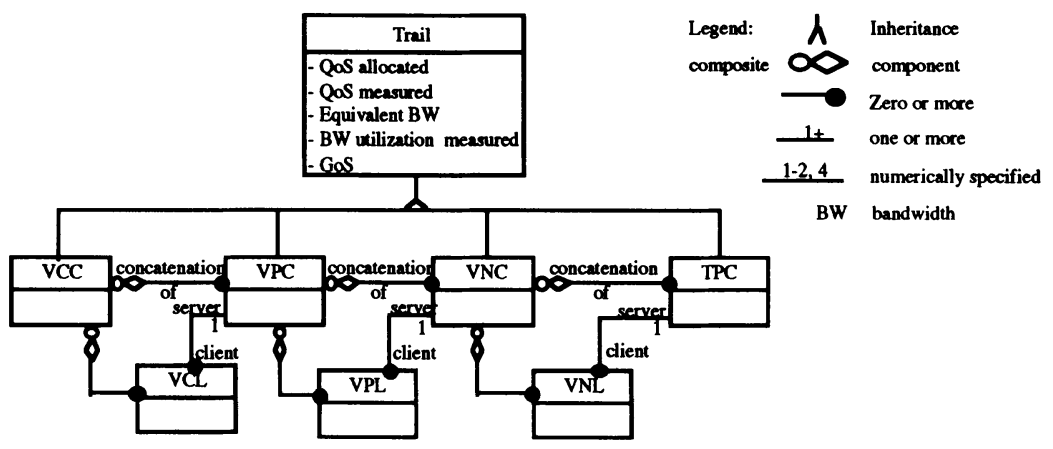

Figure 6: Client-server relationship of connection objects between adjacent layer networks.

- Objects with inter-view relationship: CTP, TTP, Subnetwork and SubnetworkConnection are the object classes that exist in both network view and element view of the information model. These objects carry the network state measurement information from individual network elements and forward it to the network view for aggregation, filtering and interpretation. In other words, these classes are the only element view aspects required to construct the network view. The CTP and TTP classes appear the same in both views. The subnetwork and the SubnetworkConnection network view object instances at their smallest granularity are equivalent to the ATMCrossConnectControl and the ATMCrossConnect at the element view respectively, as suggested by TINA's NRIM.

\section{SUMMARY}

This paper has given an overview of how Virtual Network, layer network, computational modeling and information modeling concepts can be applied for resource management within an ATM network. We have stressed the power of the layer network concept, which leads to a hierarchical structure of similar, interacting Layer Network Managers responsible for the management of their layer network state information and the allocation of their resources to other client layers where applicable. We have also introduced a Virtual Network layer, which we believe will be an important concept for the fair allocation of network resources amongst competing network services and applications. The implementation of traditionally separate traffic and network management functions within a repeating layered management structure provides a rich and flexible foundation for future resource management systems.

Such systems will likely take advantage of the advances made in the emerging distributed computing environment, e.g. CORBA. This environment will offer a platform for the flexible distribution and expansion of the management capabilities presented here, and brings with it new challenges regarding implementation for good "signaling and delay" performance, and design for scalable and stable operation. 


\section{REFERENCES}

[ATMF-v4] ATM Forum Traffic Management Working Group, Draft ATM Forum Traffic Management Specification Version 4.0, December 18, 1994.

[ATMF-M4ev] "M4 Interface Requirements and Logical MIB: ATM Network Element View", Version 1, ATM Forum, October 1994.

[ATMF-M4nv] "M4 Interface Requirements and Logical MIB: ATM Network View", Version 1 (Draft), ATM Forum, 1995.

[Dziong96] Z. Dziong, Y. Xiong and L. Mason, "Virtual Network Concept and its Applications for Resource Management in ATM Based Networks", Broadband Communications '96, Montreal, Canada, April 23-25, 1996.

[ElWa193] A. I. ElWalid and D. Mitra, "Effective bandwidth of general Markovian traffic sources and admission control of high speed networks", IEEE/ACM Trans. on Networking, vol. 1, no. 3, June 1993, pp. 329-343.

[Farago95] A. Farago, S. Blaabjerg, L. Ast, G. Gordos and T. Henk, "A New Degree of Freedom in ATM Network Dimensioning: Optimizing the Logical Configuration", IEEE JSAC, vol. 13, no. 7, pp. 1199-1206, September 1995.

[Fowler95] H. Fowler (Bellcore), "TMN-Based Broadband ATM Network Management, IEEE Communications Magazine, March 1995.

[G.805] ITU-T Recommendation G.805 (Draft), "Generic Functional Architecture of Transport Network".

[I.326] ITU-T Recommendation I.326 (Draft), "Functional Architecture of Transport Networks Based on ATM".

[1.371] ITU-T Recommendation I.371, "Traffic Control and Congestion Control in B-ISDN", March 1993.

[Kesid93] G. Kesidis, J. Walrand, C. S. Chang, "Effective bandwidths for multiclass Markov fluids and other ATM sources", IEEE/ACM Trans. on Networking, vol. 1, no. 4, August 1993, pp. 424-428.

[M.3010] ITU - CCITT Recommendation M.3010, "Principles for a Telecommunications Management Network", October 1992.

[Rumb91] J. Rumbaugh, Object-Oriented Modeling and Design, Prentice Hall, 1991.

[TA-1114] TA-NWT-001114, "Generic Requirements for Operations Interfaces Using OSI Tools: ATM/Broadband Network Management", Bellcore Technical Advisory, Issue 2, Livington, N.J., October, 1993.

[TINA95] "Overall Concepts and Principles of TINA", TINA Document TB_MDC.018_1.0_94, February 1995.

[TINA95a] "Computational Modelling Concepts", TINA Document TB_NAT.002_3.1_94, December, 1994.

[TINA95b] "Network Resource Information Model Specification", TINA Document TB_LR.010_2.0_94, December, 1994.

Biography

G. Woodruff and P. Appanna are Ph.D. students at the University of Toronto.

N. Peripanathan and F. Chang completed their M.A.Sc. degree in 1996 and are currently with Nortel.

A. Leon-Garcia is a professor in the department of electrical and computer engineering at the University of Toronto. 